\title{
A numerical MHD model for the solar tachocline with meridional flow
}

\author{
A. Sule, G. Rüdiger, and R. Arlt \\ Astrophysikalisches Institut Potsdam, An der Sternwarte 16, 14482, Potsdam, Germany \\ e-mail: gruediger@aip.de
}

Received 29 September 2004 / Accepted 10 March 2005

\begin{abstract}
There are successful approaches to explain the formation of the tachocline by a poloidal magnetic field in the solar core. We present here the first MHD simulations of the solar tachocline which self-consistently include the meridional circulation. We show that the meridional flow significantly changes the shape and the characteristics of the tachocline. We find that after the inclusion of the meridional circulation, a tachocline can be formed even when the poloidal field lines are crossing the boundary between the radiative zone and the convection zone. We also discuss the effects of the magnetic Prandtl number as well as of the magnetic Reynolds number on the properties of the tachocline. The tachocline is much thinner for higher magnetic Reynolds numbers and/or lower magnetic Prandtl numbers. We expect that a poloidal magnetic seed field of around $1 \mathrm{G}$ will be sufficient to produce the tachocline of the Sun. However, the model requires the initial magnetic field to be in a narrow range to satisfy tachocline solutions. The simulations including a stable temperature gradient produce a shallower as well as slower meridional circulation than the ones without it, as required from the lithium abundance at the solar surface.
\end{abstract}

Key words. magnetohydrodynamics (MHD) - Sun: interior - Sun: magnetic fields - Sun: rotation

\section{Introduction}

The term tachocline refers to a very thin layer at the base of the convection zone which separates the latitudinal differential rotation inside the convection zone from the uniformly rotating radiative zone. Current observations put the position of its center at around $0.69 R_{\odot}$ and estimate its thickness to be slightly less than $0.05 R_{\odot}$. There is also a general belief that the tachocline is prolate and is also thicker near the pole. However, the observations for higher latitudes have large error bars, and hence the evidence for the latitudinal dependence of the tachocline is not yet conclusive (Schou et al. 1998; Antia et al. 1998; Charbonneau et al. 1999; Basu \& Antia 2001). The facts were verified qualitatively in frequency-versus-radius plots presented by Eff-Darwich et al. (2002) and Howe (2003).

There have been many attempts to explain the formation of such a thin shear layer inside the Sun. Spiegel \& Zahn (1992) were the first to give a purely hydrodynamic model for the tachocline. Elliott (1997) extended this analysis numerically also including meridional circulation. These models were successful in explaining the stability of the tachocline using anisotropic turbulence but the equilibrium thickness of the tachocline obtained was much thicker than latest observational estimates. Also later works such as that of Miesch (2003) confirm that a purely hydrodynamic model is insufficient to explain the tachocline.
Rüdiger \& Kitchatinov (1997) studied the tachocline under the influence of a magnetic field. They believed that the existence of a weak seed field of about 0.01 to $1 \mathrm{mG}$ in the solar core is highly likely and this weak internal magnetic field will be mainly responsible for the formation of the tachocline. Several other authors like Gough \& McIntyre (1998), MacGregor \& Charbonneau (1999), and Garaud (2002) have used this idea of a weak poloidal seed field forming the tachocline.

Here we present a magnetohydrodynamic model that selfconsistently calculates the meridional flow. In this work, we show how the inclusion of the meridional circulation changes the shape of the tachocline and discuss the effects of varying magnetic Prandtl number and magnetic Reynolds number on the structure and the properties of the tachocline. Finally, we discuss the effect of the stable temperature gradient on the structure of the meridional flow.

For a very simple magnetic shear flow model, the relation

$\frac{\delta R}{R} \propto \mathrm{Ha}^{-1 / 2}$

has been derived by Rüdiger \& Kitchatinov (1997) for the fractional thickness of any magnetically induced tachocline layer with the Hartmann number

$\mathrm{Ha}=\frac{B_{0} R}{\sqrt{\mu_{0} \rho v \eta}}$ 
implying that rather thin tachoclines of say $0.03 R_{\odot}$ always require $\mathrm{Ha} \simeq 10^{3}$. The Hartmann number also appears in the relation between the induced toroidal magnetic field $B_{\phi}$ and the given poloidal field amplitude $B_{0}$, i.e.

$\frac{B_{\phi}}{B_{0}} \simeq \frac{\Omega_{0} R}{B_{0}}\left(\frac{\mu_{0} \rho v}{\eta}\right)^{0.5} \simeq \frac{\mathrm{Rm}}{\mathrm{Ha}}$

(the amplification factor), with the global magnetic Reynolds number $\mathrm{Rm}=\Omega_{0} R^{2} / \eta$.

With $\eta \simeq 10^{3} \mathrm{~cm}^{2} / \mathrm{s}$ as a rough estimate for the solar diffusivity, one finds $\mathrm{Rm} \simeq 10^{12}$ for the solar transition region between the convection zone and the radiative interior so that a very strong amplification of nine orders of magnitude should occur. The code used below only reaches Rm-values of order $10^{5}$, thus being limited in the amplification factors.

Forgács-Dajka \& Petrovay (2002) and Petrovay (2003) suggested that the tachocline, being just below the base of the convection zone, is more likely to be turbulent and hence the values of the diffusivities in this region are more likely to be as high as those in the convection zone. Studies by several authors concentrated on the stability of the tachocline being subject to latitudinal shear (Watson 1981; Gilman \& Fox 1997; Garaud 2001; Cally 2003; Dikpati et al. 2003). These ideas, however, are beyond the scope of the present discussion.

\section{The model}

The principle aim of this work will be to reproduce the observed rotation profile in the tachocline region, along with the nearly uniform rotation in the solar radiative zone below.

In order to be consistent with the observations, we require that (i) the thickness of the tachocline should be less than $0.05 R_{\odot}$; (ii) the core should settle near uniform rotation; (iii) the meridional circulation should be slow and/or should not penetrate the radiative zone deeply; and (iv) the internal seed field should be weak.

We assume that the tachocline lies completely inside the radiative zone. The equations take an axisymmetric, incompressible Boussinesq form. Although we would like to include density stratification for more realistic simulations, our test runs with even moderate density gradients proved computationally too expensive. We take the outer radius of the computational domain as $R_{\text {out }}=0.75 R_{\odot}$ and the inner radius as $R_{\text {in }}=0.1 R_{\odot}$. Due to numerical constraints, we restrict ourselves to spherical shells instead of a complete sphere. The size of the remaining inner hole was found to have a negligible effect on the results.

We also assume that the rotation profile in the convection zone is independent of the dynamics in the radiative zone and can be prescribed as a boundary condition. We further assume that any physical phenomenon occurring in the convection zone, including a dynamo, does not have an effect on the dynamics within the radiative zone and the tachocline. For the first set of simulations, the effect of the temperature gradient and hence buoyancy force on the fluid is also neglected. We will present the equations and results involving the temperature in Sect. 5.
The magnetohydrodynamic equations employed are

$$
\frac{\partial \boldsymbol{u}}{\partial t}=-(\boldsymbol{u} \cdot \nabla) \boldsymbol{u}-\nabla P+v \Delta \boldsymbol{u}+\frac{1}{\mu_{0} \rho}(\nabla \times \boldsymbol{B}) \times \boldsymbol{B},
$$

$\frac{\partial B_{\phi}}{\partial t}=[\nabla \times(\boldsymbol{u} \times \boldsymbol{B})]_{\phi}-\eta[\nabla \times(\nabla \times \boldsymbol{B})]_{\phi}$

with $\nabla \cdot \boldsymbol{u}=0$ and $\nabla \cdot \boldsymbol{B}=0$ where $v$ and $\eta$ are the constant viscosity and magnetic diffusivity, respectively. Other symbols have their usual meaning. The poloidal magnetic field is maintained time-invariant. This approximation is valid as in the Sun, due to the small $\eta$, the magnetic diffusion time is believed to be several time larger than the present solar age, whereas the stable tachocline solution is reached in our simulations in much shorter times.

The equations are solved in a spherical shell geometry using the spectral code developed by Hollerbach (1994) and Hollerbach (2000). The equations are scaled in time by the magnetic diffusion time, $\tau_{\text {diff }}=R_{\text {out }}^{2} / \eta$, in velocity by $\eta / R_{\text {out }}$, and the magnetic field is normalized by $B_{0}$, thus generating the dimensionless Lundquist number

$S_{0}=\frac{B_{0} R_{\text {out }}}{\sqrt{\mu_{0} \rho} \eta}$

This reduces the equations to very few free parameters, namely $r_{\text {out }}=R_{\text {out }} / R_{\odot}, r_{\text {in }}=R_{\text {in }} / R_{\odot}, \mathrm{Rm}, \mathrm{Pm}=v / \eta$ and $S_{0}$.

Given the very high turbulent viscosity in the convection zone, we can safely assume that the convection zone rotation profile acts like a rigid outer boundary on the tachocline. Therefore, the rotation profile of the angular velocity at the outer boundary is chosen to match the observed rotation profile in the bulk of the convection zone, which is also used by MacGregor \& Charbonneau (1999) and is maintained as a rigid boundary condition. It is given by

$\Omega_{\text {out }}=\Omega_{0}\left(1-0.1264 \cos ^{2} \theta-0.1591 \cos ^{4} \theta\right)$

where $\theta$ is the co-latitude ${ }^{1}$. The remaining boundary conditions on the velocity are maintained stress-free. For the magnetic field, we set vacuum boundary conditions at the inner as well as the outer boundary.

Due to computational limitations, we will use values of $\mathrm{Rm}$ not exceeding $10^{5}$. We assume that the magnetic diffusivity $\eta$ and the viscosity $v$ take their molecular values in the tachocline region, but our numerically constrained choice of Rm implies very high values of $\eta$ and $v$ in the simulations.

The computations evolve only the axisymmetric modes of the originally three-dimensional spectral code. The runs typically employ 60 radial Chebyshev $(k)$ modes and 60 latitudinal Legendre $(l)$ modes. It has been verified by additional computations that the results change very little at different resolutions. The Chebyshev polynomials are ideal to resolve the radial boundary layers very well.

\footnotetext{
1 MacGregor \& Charbonneau (1999) give a positive sign for the $\cos ^{4} \theta$ term which appears to be a typo. The negative sign used here is essential for a solar-like differential rotation.
} 
The magnetic field structure used for the internal seed field is dipolar with no initial toroidal field i.e.

$\boldsymbol{B}=\left(\frac{1}{r^{2} \sin \theta} \frac{\partial A}{\partial \theta},-\frac{1}{r \sin \theta} \frac{\partial A}{\partial r}, 0\right)$

with the generating function

$A=S_{0} r^{2}\left(1-\frac{r}{r_{\text {out }}}\right)\left(1-\frac{r_{\text {in }}}{r}\right) \sin ^{2} \theta$.

The function involves the second bracket in order to avoid the magnetic field lines crossing the inner boundary. This will introduce small curls of the poloidal magnetic field near the inner boundary. We eliminate the curl of the poloidal magnetic field, which is dominant only at the inner boundary, by equating it to zero at every time step. A comparison with computations including the full Lorentz force did not show significantly different results.

\section{The effect of the meridional flow}

Earlier investigations have shown that even a weak poloidal seed field is able to produce a solar-like tachocline. It was found that the region near the rotation axis (i.e. poles) is least affected by the field and the tachocline is thickest in that part. Further, when the poloidal field amplitude is very small, the magnetic field is unable to alter the rotation, and the resulting rotation profile looks very similar to the non-magnetic case. At the other extreme, if the magnetic field strength is very high, the contour lines of constant $\Omega$ follow the poloidal magnetic field lines throughout the interior, in accordance with the theorem of Ferraro (1937). A solar-like tachocline is thus impossible (Garaud 2002) in either case. Our first simulations neglecting the meridional flow concur with most of these key results.

In the simulations of this section, we use $\mathrm{Rm}=10^{4}$ and $\mathrm{Pm}=1$. In all our simulations, we found that the system converges to an equilibrium solution in less than $0.1 \tau_{\text {diff }}$. Unless mentioned otherwise, the figures and numerical values throughout this Paper refer to the final equilibrium solutions. The results are best represented by the comparison of the following two graphs. While the left panel of Fig. 1 is just a reproduction of the earlier MacGregor \& Charbonneau (1999) result, where the magnetic fields are confined to the simulation domain, the right panel of Fig. 1 shows the same model including the meridional circulation. The solid lines are the iso-rotation curves whereas the dashed and the dotted lines represent the contours of the positive and negative toroidal field strength, respectively. While in the left graph the region along the rotation axis is not affected at all, we see that the inclusion of the meridional circulation changes the picture completely.

We observe that the entire core, including the region near the rotation axis, has achieved nearly uniform rotation. The tachocline is formed near the outer boundary. In contrast with the results ignoring the meridional flow, the tachocline is now thinnest near the pole. In the region near the equator, where the magnetic field influence is smaller, we find that the iso-rotation curves tend to be similar to the characteristic Taylor-Proudman flow. We further observed that the toroidal magnetic field strength is only $30 \%$ of the poloidal magnetic field strength.
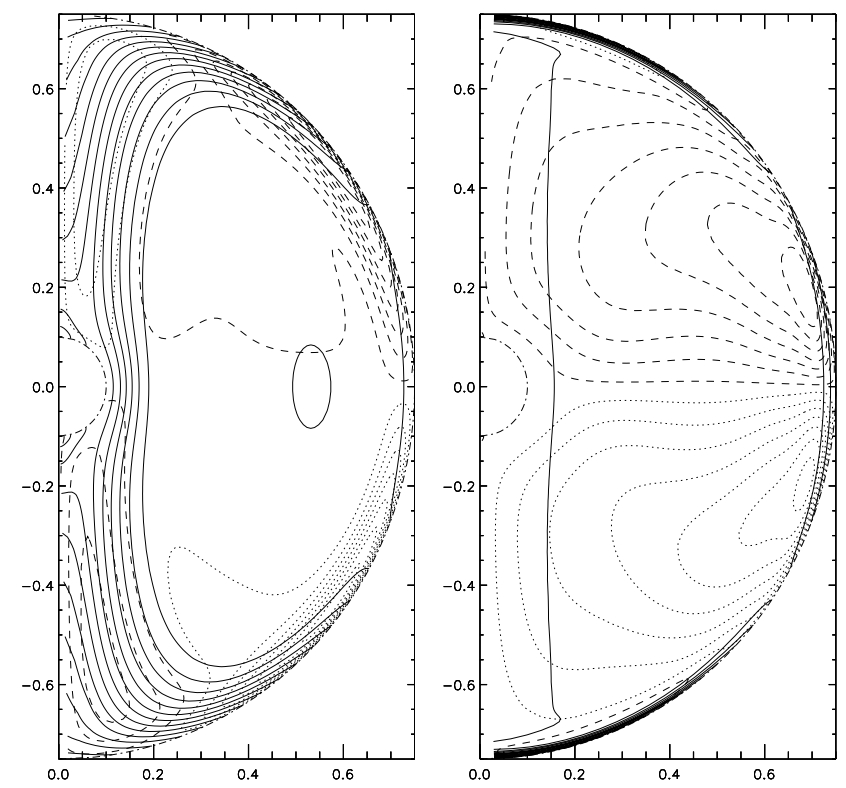

Fig. 1. Results for the simulations excluding (left) and including (right) the meridional circulation with the magnetic field being confined to the core. The solid lines are the iso-rotation curves, whereas the dashed and the dotted lines represent the contours of the positive and the negative toroidal field strength respectively. The dot-dashed lines indicate the boundaries of the simulation domain. The timeinvariant poloidal magnetic field is not shown. $S_{0}=1100, \mathrm{Pm}=1$ and $\mathrm{Rm}=10^{4}$.
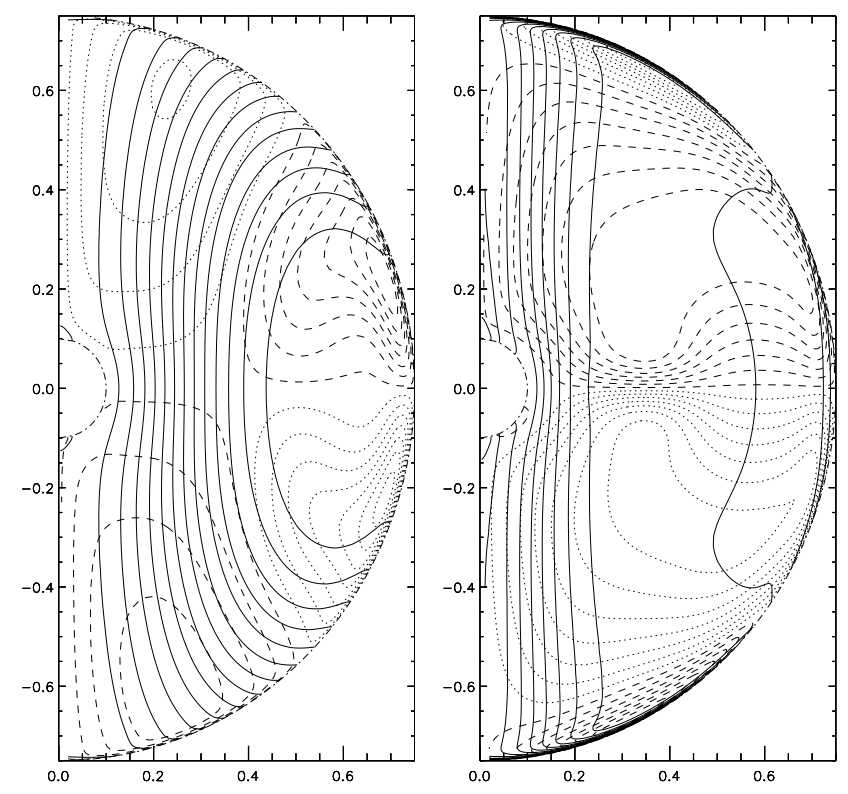

Fig. 2. Results for the simulations excluding (left) and including (right) the meridional circulation when the magnetic field lines are crossing the outer boundary.

Following MacGregor \& Charbonneau (1999), we also studied the second case, where the poloidal magnetic field is no longer confined to the computational domain and the magnetic field lines are crossing the outer boundary of the simulation domain. While this case produced no real tachocline for the MacGregor \& Charbonneau (1999) model, this is no longer a problem after the inclusion of the meridional flow (see Fig. 2). 

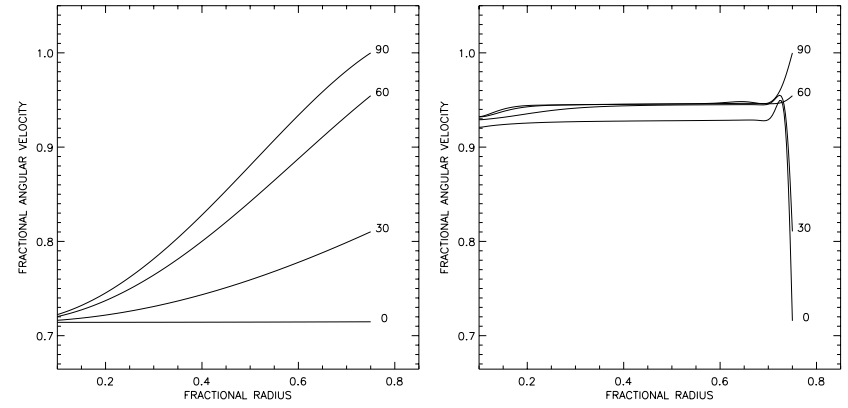

Fig. 3. Fractional $\Omega$ vs. fractional radius for the initial configuration (left) and the equilibrium solution when $B_{\text {pol }}$ is constant (right). Different lines represent $\Omega$ at different co-latitudes.
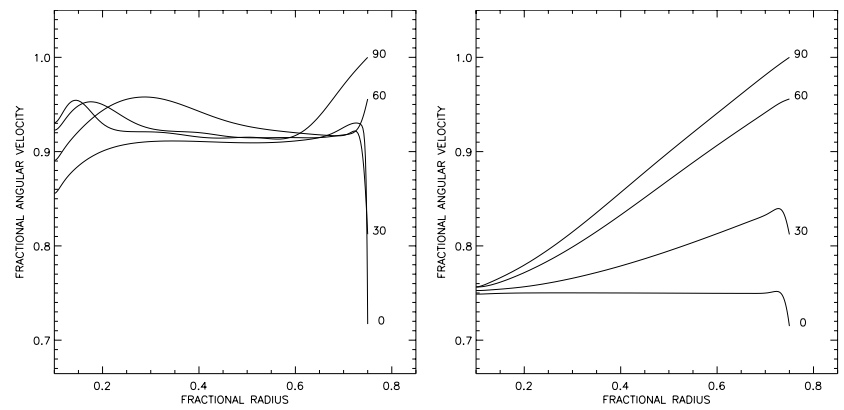

Fig. 4. Fractional $\Omega$ vs. fractional radius for a snapshot at $0.05 \tau_{\text {diff }}$ (left) and $0.5 \tau_{\mathrm{diff}}$ (right) with decaying $B_{\mathrm{pol}}$. Again, different lines represent $\Omega$ at different co-latitudes.

The initial and final states in terms of fractional $\Omega$ are shown as functions over radius for various latitudes in Fig. 3 .

We performed some test simulations with a decaying poloidal magnetic field. During these simulations, we observed that the magnetic field does form a tachocline during the period up to $\sim 0.05 \tau_{\text {diff }}$, but as the magnetic field decays gradually due to the high value of $\eta$ assumed in the simulations, the flows readjust themselves, and the resulting pattern will be the same as the purely hydrodynamic case, i.e. it will approach the Taylor-Proudman flow. Figure 4 shows the fractional $\Omega$ in an intermediate stage at $t=0.05 \tau_{\text {diff }}$ for a decaying poloidal magnetic field, keeping other parameters the same as for the run in Fig. 3. The effect of forming a tachocline structure by a time-dependent poloidal field is thus very similar to the non-decaying field, but diffusivity reduces the field unnaturally early, and the final tachocline state is not achieved. Figure 5 shows the evolution of the poloidal magnetic field at three different times.

We conclude here that the magnetic field is not only important for the formation of the tachocline but is also important for maintaining it, at least in the case of the high magnetic diffusivity used in these simulations.

\section{Effect of varying magnetic Prandtl number and magnetic Reynolds number}

\subsection{Varying the magnetic Prandtl number}

Following Kippenhahn \& Weigert (1994) and Stix \& Skaley (1990), the solar value for the magnetic Prandtl number is of
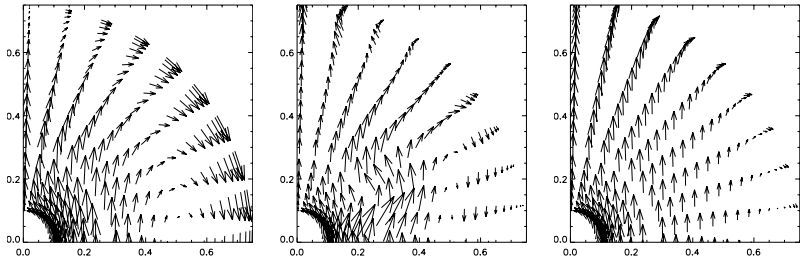

Fig. 5. Snapshots of $B_{\text {pol }}$ in case of a decaying poloidal field at (from left to right) $t=0,0.05 \tau_{\mathrm{diff}}$, and $0.5 \tau_{\mathrm{diff}}$. For each snapshot, the length of the arrows is proportional to the amplitude of $B_{\mathrm{pol}}$ at each point. The length of the arrows is renormalized in each plot by corresponding maximum field amplitude. Only every fifth arrow in $\theta$ and $r$ is plotted in order to avoid congestion.
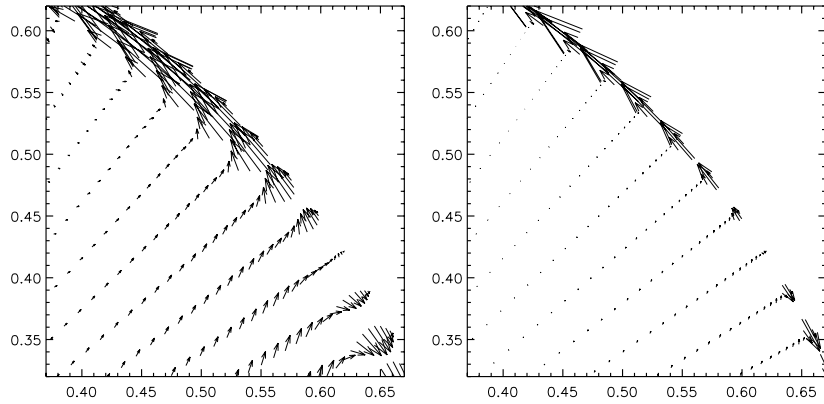

Fig. 6. Results for the simulations with $\mathrm{Pm}=1$ (left) and 0.05 (right) showing a small section of the simulation domain with the components of the meridional circulation plotted. The arrow lengths are assigned in the same way as Fig. 5. Only alternate points in the radial direction are plotted.

the order of $10^{-3}$. Although this value was not achieved, the simulations were performed for various values of $\mathrm{Pm}$ in the range of $0.05-1$ whereas $\mathrm{Rm}=10^{4}$ in all the cases.

In these simulations, we observed that the horizontal component of the meridional flow $\left(u_{\theta}\right)$ generated is about $2 \%$ of $u_{\phi}$ at higher latitudes and is about $1 \%$ for lower latitudes. Similarly, the vertical component of the meridional flow $\left(u_{r}\right)$ is about $0.5 \%$ (see Fig. 7 left). These ratios are nearly independent of the value of Pm. The radial component of the velocity remains much smaller than the latitudinal component especially for lower values of Pm, as can be seen in Fig. 6. The figure also shows that strong latitudinal flows are expelled closer to the outer boundary for lower values of Pm. The tachocline, thus formed, is thinner ${ }^{2}$ at the pole than at the equator. The thickness of the tachocline at the equator reduces marginally from $0.030 R_{\odot}$ to $0.026 R_{\odot}$ when Pm is changed from 1 to 0.05 , whereas the thickness of the tachocline at the pole decreases considerably. We also observe that the amplitude of the toroidal magnetic field remains smaller than that of the poloidal field and is almost independent of the choice of Pm.

\subsection{Varying the magnetic Reynolds number}

Now we will use a constant $\mathrm{Pm}=1$ and vary $\mathrm{Rm}$. As we know that the solar value of $\mathrm{Rm}$ is about $10^{12}$, we ran simulations

2 The thickness is defined as the distance between the outer boundary and the radius at which the rotation rate deviates by $1 \%$ from the rotation rate deep inside the core. 

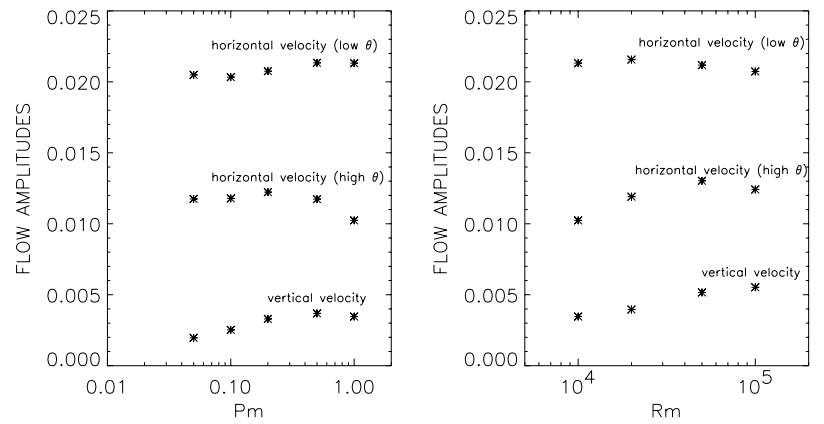

Fig. 7. The amplitudes of the components of the meridional flow at different co-latitudes $(\theta)$, normalized to the rotational velocity at the equator, with varying $\mathrm{Pm}$ (left) and $\mathrm{Rm}(r i g h t)$.

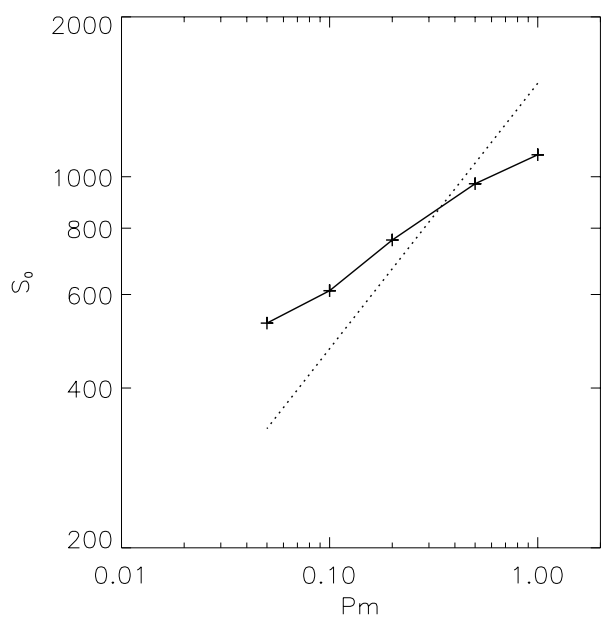

Fig. 8. Variation of $S_{0}$ with varying $\mathrm{Pm}$ as a $\log -\log$ plot, for an approximate tachocline thickness of $0.025 R_{\odot}$. The dotted line represents a fixed $\mathrm{Ha}=1500$.

for $\mathrm{Rm}$ larger than $\mathrm{Rm}=10^{4}$. The highest value of $\mathrm{Rm}$ in our simulations was $10^{5}$.

In this set of simulations we again notice that the relative amplitudes of the meridional flow are nearly independent of the value of Rm as shown in Fig. 7 (right). The thickness of the tachocline at the equator reduces from $0.030 R_{\odot}$ to $0.015 R_{\odot}$ when $\mathrm{Rm}$ is changed from $10^{4}$ to $10^{5}$, whereas the thickness of the tachocline at the pole goes down from $0.012 R_{\odot}$ to $0.003 R_{\odot}$. Correspondingly, the toroidal field $\left(B_{\phi}\right)$ in the tachocline region increases from $0.3 B_{0}$ to $1.3 B_{0}$.

\subsection{Effect on the Lundquist number}

It will be worthwhile to place some emphasis on the choice of the poloidal field strength. In our simulations, we find that the choice of the amplitude of the seed field value is very critical. A small deviation in either direction from the magnetic field which produces a solar-like tachocline either makes the iso-rotation curves similar to the non-magnetic case or the curves will comply with Ferraro's theorem, unable to produce a tachocline in both cases. At lower values of Pm, we require smaller seed field as shown in Fig. 8. At higher values of Rm, the required value of $S_{0}$ is higher (Fig. 9).

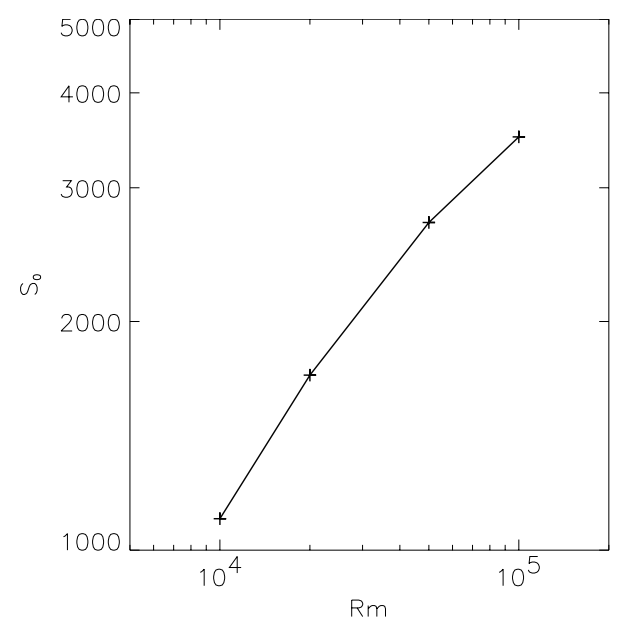

Fig. 9. Variation of $S_{0}$ with varying $\mathrm{Rm}$ as a $\log -\log$ plot, for an approximate tachocline thickness of $0.028 R_{\odot}$.

An empirical law governing the variation of $S_{0}$ as a combined function of $\mathrm{Pm}$ and $\mathrm{Rm}$ can be deduced from these plots as

$S_{0} \simeq 10 \mathrm{Rm}^{0.5} \mathrm{Pm}^{0.25}$.

This scaling is the main result of the computations. It means that

$$
\frac{B_{0}}{\sqrt{\mu_{0} \rho}} \simeq 10 \sqrt[4]{\Omega^{2} v \eta}
$$

which, of course, has the correct dimension of a velocity. The old estimate without meridional flow of Rüdiger \& Kitchatinov (1997) led to the quite different expression $B_{0} / \sqrt{\mu_{0} \rho} \simeq$ $10^{3} \sqrt{v \eta} / R$ which is a very small value due to the appearance of the radius $R$. Equation (11) yields

$\frac{B_{0}}{\sqrt{\mu_{0} \rho}} \simeq 0.1 \mathrm{~cm} / \mathrm{s}$

so that a maximum field amplitude of 1 Gauss results, for an average density of $10 \mathrm{~g} / \mathrm{cm}^{3}$. This is a much larger value than the mGauss values for models without meridional flow, but it is not an unrealistic number. In contrast to the old model, the toroidal field belts now have the same order of magnitude as the poloidal fields.

\section{Effect of a temperature gradient}

In the simulations discussed in the previous sections, we noted that the amplitude of the meridional circulation was nearly independent of the variation of $\mathrm{Rm}$ as well as Pm. In the lower latitudinal belt, where the solar dynamo is likely to be located, the amplitude of horizontal velocity $u_{\theta}$ was always around $1 \%$ of $u_{\phi}$, and the flow reached very deep layers of the shell. The lithium abundance, however, as observed at the solar surface suggests that the meridional circulation should either be very shallow or very slow. Otherwise lithium would be destroyed in its fusion zone below $0.68 R_{\odot}$.

We therefore include a given temperature gradient in our model. We introduce a temperature fluctuation $\Theta$ on top of this 

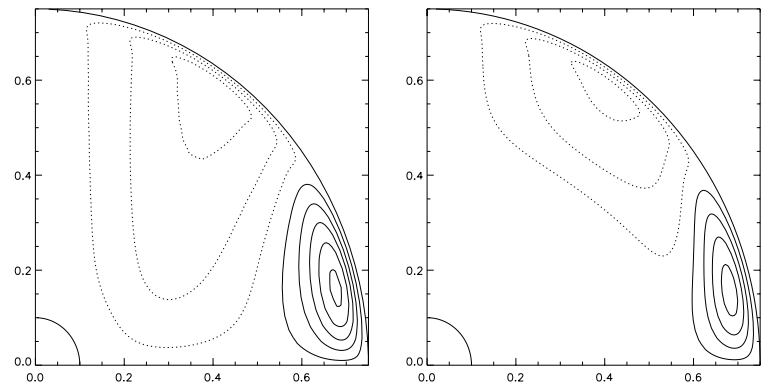

Fig. 10. Results for the simulations excluding (left) and including (right) buoyancy. The solid and the dotted lines are clockwise and anti-clockwise meridional circulation, respectively. $\tilde{\mathrm{R}} \mathrm{a}=-2 \times 10^{7}$, $\mathrm{Rm}=10^{4}$.

temperature profile. This fluctuation induces a buoyancy force. Hence Eq. (4) will be modified to read

$$
\frac{\partial \boldsymbol{u}}{\partial t}=-(\boldsymbol{u} \cdot \nabla) \boldsymbol{u}-\nabla P+v \Delta \boldsymbol{u}+\frac{1}{\mu_{0} \rho}(\nabla \times \boldsymbol{B}) \times \boldsymbol{B}+\boldsymbol{g} \rho,
$$

where $\boldsymbol{g}$ is the vector of gravitational acceleration. In the context of the Boussinesq approximation and after rescaling as in Sect. 2, we obtain a buoyancy force as $\tilde{R} \mathrm{a} \Theta r$ with the modified Rayleigh number

$\tilde{\mathrm{R}} \mathrm{a}=\frac{g \alpha \Delta T R_{\mathrm{out}}^{3}}{\eta^{2}}$,

where $\Delta T=T_{\text {in }}-T_{\text {out }}$ and $\alpha$ is the coefficient of volume expansion. The equilibrium solution obtained from the temperature equation alone is chosen as the mean temperature profile (normalized with its bottom value) and is given by

$T_{0}=\frac{r_{\text {in }}}{r_{\text {out }}-r_{\text {in }}}\left(\frac{r_{\text {out }}}{r}-1\right)$

and the non-dimensional energy equation has the form

$\frac{\partial \Theta}{\partial t}=\frac{\operatorname{Pm}}{\operatorname{Pr}} \Delta \Theta-\boldsymbol{u} \cdot \nabla\left(\Theta+T_{0}\right)$,

where $\operatorname{Pr}=v / \chi$ is the Prandtl number using the thermal diffusivity $\chi$.

When the model is evolved from the initial state including the temperature equation, it takes much longer (in terms of magnetic diffusion times) to achieve a steady state solution than the simpler cases in the previous sections. To save computational resources, we used the steady state solutions obtained in the previous sections as the initial condition for the simulations involving temperatures. It was verified that the solutions obtained in this manner are identical to the solutions obtained from same buoyancy runs but starting with the initial conditions of Sect. 2 .

We used $\mathrm{Rm}=10^{4}$ and $\mathrm{Pm}=1$ for these sets of simulations. As the simulation domain is of radiative nature, we use negative values of $\tilde{R}$. We performed the simulations with various values of $\tilde{R}$. The results are presented in Fig. 10. For the regime when $-\tilde{R} a / \mathrm{Rm}^{2} \leq 0.01$, the buoyancy is unable to produce any major change in any of the velocity components in magnitude or in structure. For more negative Ra,

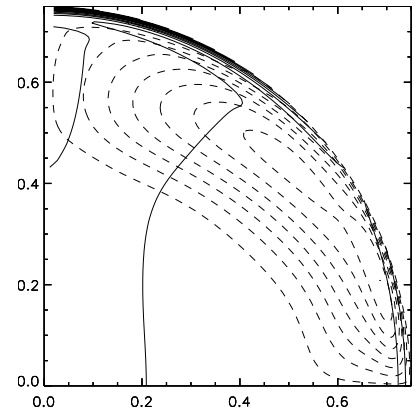

Fig. 11. The same as in Fig. 1 but with the effect of buoyancy, $\mathrm{Ra}=-2 \times 10^{7}, \mathrm{Rm}=10^{4}$.

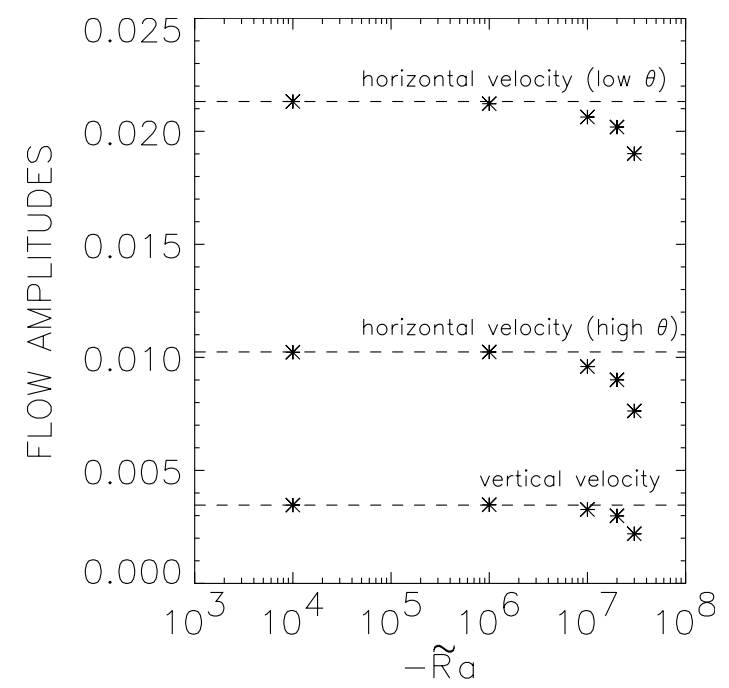

Fig. 12. Plot of the amplitudes of the components of the meridional flow at different co-latitudes $\theta$, normalized to the rotational velocity at the equator, with varying $\tilde{R} a$. The dashed lines represent the corresponding values without buoyancy effect. $\mathrm{Rm}=10^{4}, \mathrm{Pm}=1$.

we see a gradual change in the structure of the meridional circulation $^{3}$. The circulation is then much shallower for higher latitudes (low $\theta$ ), as desired to explain weak mixing into the interior. The magnitude of the flow also decreases but the change is not drastic; see Fig. 12. For the lower latitudinal belt (high $\theta$ ), which is an important region for the solar dynamo, the decrease is relatively stronger and the depth of the circulation is also clearly reduced. We obtain a marginal increase in the amplification of the toroidal field, probably because the field is not advected through the entire computational domain any longer. The structure of the toroidal field is thus changed as well, and it is shifted towards the outer parts of the shell. On the other hand, the rotation rate at higher latitudes, at large depth in the core, becomes slightly slower than that at the equator. But even this change is marginal and within the observational limits $\left(\left|1-\Omega_{\text {pole }} / \Omega_{\text {equator }}\right| \leq 3 \%\right.$ for $\left.r \leq 0.65 R_{\odot}\right)$. Angular velocity and toroidal field belts are shown in Fig. 11. We expect that even more negative $\tilde{R}$ a will further reduce the depth and amplitude of the meridional circulation.

\footnotetext{
${ }^{3}$ Note that $-\tilde{\mathrm{R}} \mathrm{a} / \mathrm{Rm}^{2} \simeq g /\left(\Omega^{2} R\right)$.
} 


\section{Summary}

In this work, we have presented the first MHD model for the solar tachocline which self-consistently calculates the meridional circulation. The consideration of the meridional flow changes the shape, the structure and the characteristics of the tachocline radically. Hence, the meridional circulation cannot be neglected while modeling the solar tachocline. The thickness of the tachocline in the outer boundary layer near the equator will be determined by the gradient of the magnetic field near the boundary. Our simulations show that the tachocline is thinner near the pole. But the simulation domain has uniform values of viscosity and magnetic diffusivity, hence we simulate only that part of the tachocline, which is inside the radiative zone. Hence, a definitive conclusion about the thickness of the tachocline in the polar region cannot be drawn from these simulations.

The tachocline is thinner at lower values of Pm as well as at higher values of $\mathrm{Rm}$. The meridional circulation is nearly independent of the variation of $\mathrm{Rm}$ and $\mathrm{Pm}$. The amplification of the toroidal magnetic field is naturally larger at higher values of $\mathrm{Rm}$, whereas from (11) it is clear that the poloidal magnetic field amplitude required to produce a solar-like tachocline goes down with decreasing $\eta$, i.e. increasing $\mathrm{Rm}$. The magnetic seed field required to produce a solar-like tachocline is a function of $\mathrm{Rm}$ as well as Pm. The value of the seed field is expected to be around 1 Gauss in the Sun, for an average density of $10 \mathrm{~g} / \mathrm{cm}^{3}$. Again a significant change is noted from the simulations without meridional flow where even a sub-mGauss field was enough to produce a solar-like tachocline. The scaling of the magnetic field as a function of the rotation rate, $v$, and $\eta$ is given in (11). The toroidal magnetic field is expected to be a few orders higher than the poloidal seed field in the case of the Sun.

When a stable temperature gradient is introduced across the shell, it makes the meridional circulation shallower as well as weaker for stronger stabilization (more negative Rayleigh number). This can prevent lithium from reaching its fusion zone which starts just below tachocline. The stabilizing effect is in line with the relatively high lithium abundance observed at the surface of the Sun. The toroidal magnetic field in this case is limited to belt in the outer parts of the radiative zone.
The relations describing the variation of various parameters such as the Lundquist number required to form a solar-like tachocline, the amplification of the toroidal magnetic field, the amplitude of the meridional circulation etc. are based on the simulation results for a limited range of $\mathrm{Rm}$ and $\mathrm{Pm}$. We hope to improve the code and verify the correctness of these relations closer to the solar values of $\mathrm{Rm}$ and $\mathrm{Pm}$ in the near future.

Acknowledgements. L. Kitchatinov is acknowledged for discussions about the simulation results and H. M. Antia for clarifying the observational results. J.-P. Zahn is acknowledged for going through the manuscript and giving valuable suggestions.

\section{References}

Antia, H. M., Basu, S., \& Chitre, S. M. 1998, MNRAS, 298, 543

Basu, S., \& Antia, H. M. 2001, MNRAS, 324, 498

Cally, P. S. 2003, MNRAS, 339, 957

Charbonneau, P., Christensen-Dalsgaard, J., Henning, R., et al. 1999, ApJ, 527, 445

Dikpati, M., Gilman, P., \& Rempel, M. 2003, ApJ, 596, 680

Eff-Darwich, A., Korzennik, S. G., \& Jiménez-Reyes, S. J. 2002, ApJ, 573,680

Elliott, J. R. 1997, A\&A, 327, 1222

Ferraro, V. C. A. 1937, MNRAS, 97, 458

Forgács-Dajka, E., \& Petrovay, K. 2002, A\&A, 389, 629

Garaud, P. 2001, MNRAS, 324, 68

Garaud, P. 2002, MNRAS, 329, 1

Gilman, P., \& Fox, P. 1997, ApJ, 484, 439

Gough, D. O., \& McIntyre, M. E. 1998, Nature, 394, 755

Hollerbach, R. 1994, Phys. Fluids, 6, 2540

Hollerbach, R. 2000, Int. J. Numer. Meth. Fluids, 32, 773

Howe, R. 2003, Proc. SOHO 12/ GONG+ 2002, 81

Kippenhahn, R., \& Weigert, A. 1994, Stellar Structure and Evolution (Springer)

MacGregor, K. B., \& Charbonneau, P. 1999, ApJ, 519, 911

Miesch, M. 2003, ApJ, 586, 663

Petrovay, K. 2003, Sol. Phys., 215, 17

Rüdiger, G., \& Kitchatinov, L. 1997, Astron. Nachr., 318, 273

Schou, J., Antia, H. M., Basu, S., et al. 1998, ApJ, 505, 390

Spiegel, E. A., \& Zahn, J.-P. 1992, A\&A, 265, 106

Stix, M., \& Skaley, D. 1990, A\&A, 232, 234

Watson, M., Geophys. Astrophys. Fluid Dyn., 161, 285 